\title{
Adicción a Internet, videojuegos y teléfonos móviles en niños y adolescentes: Un estudio de casos y controles
}

\section{Internet, video game and mobile phone addiction in children and adolescents diagnosed with ADHD: A case-control study}

\author{
A. Menéndez-García*, A. Jiménez-Arroyo*, M. Rodrigo-Yanguas****, M. Marín-Vila**, \\ F. SÁnchez-Sánchez***, E. Roman-Riechmann*,****, H. Blasco-Fontecilla*,**,************. \\ * Universidad Autónoma de Madrid (UAM), Madrid, España. \\ ** Departamento de Psiquiatría, IDIPHIPSA-Hospital Universitario Puerta de Hierro, Majadahonda, España. \\ *** Departamento I+D+I, TEA Ediciones, Madrid, España. \\ **** Departamento de Pediatría, IDIPHIPSA-Hospital Universitario Puerta de Hierro, Majadahonda, España. \\ ***** Centro de Investigación Biomédica en Red Salud Mental (CIBERSAM), Madrid, España. \\ ****** ITA Salud Mental, Madrid, España.
}

\section{Resumen}

El uso de las nuevas tecnologías se ha generalizado a nivel mundial. Hay una creciente preocupación respecto del «trastorno de adicción a Internet» (TAI), el «trastorno de juego en Internet» (TJI) y la «adicción al teléfono móvil» (ATM). El trastorno por el déficit de atención con hiperactividad (TDAH) se ha asociado con el TAI y el TJI. Sin embargo, falta evidencia sobre la relación entre el TDAH y la ATM. Estudio naturalista de casos y controles. Comparación de 112 pacientes (51 niños con el TDAH y 61 niños sin el TDAH) con edades entre 7-17 años respecto del TAI, el TJI y la ATM. Utilizamos el cuestionario de TEA para evaluar la función ejecutiva y el TDAH (ATENTO) y el cuestionario ADITEC para obtener información diferenciada por género para el TAI, el TJI y la ATM. Las niñas obtuvieron puntuaciones más altas en la ATM (desviación típica \pm media, $D T \pm M)(25,93 \pm 17,64$ vs. $14,77 \pm$ $19,43, \mathrm{p}=, 03)$, mientras que los niños obtuvieron puntuaciones más altas en el TJI $\left(30,09 \pm 21,65\right.$ vs. $\left.12,51 \pm 16,61, \mathrm{p}<10^{-3}\right)$. Hubo una correlación moderada entre la gravedad de hiperactividad/impulsividad y el TJI $(r=, 349, p=, 013)$, pero la correlación desapareció después de controlar por el impacto en el ámbito social según las mediciones del cuestionario ATENTO $(\mathrm{r}=171, \mathrm{p}=, 250)$. A la mayoría de los padres les preocupa que sus hijos puedan tener el TAI, el TJI o la ATM. El género femenino está asociado con la ATM, mientras que el género masculino está asociado con el TJI. El TDAH es un factor de riesgo para el desarrollo del TAI y el TJI. El TDAH del tipo combinado y de predominio de hiperactividad/impulsividad están asociados con el TJI. Un buen ajuste social protege contra el desarrollo del TJI. Hay vulnerabilidades de género para el TAI, el TJI y la ATM. El TDAH es un factor de riesgo para el TJI, pero un buen ajuste social amortigua esta asociación. Palabras clave: TDAH; trastorno por adicción a Internet; trastorno de juego en Internet; adicción al teléfono móvil.

\section{Abstract}

The use of new technologies has become widespread worldwide. There is increasing concern about "Internet addiction disorder" (IAD), "Internet gaming disorder" (IGD), and "Mobile phone addiction" (MPA). Attention Deficit Hyperactivity Disorder (ADHD) has been associated with IAD and IGD. However, evidence is lacking about the relationship between ADHD and MPA. Naturalistic case-control study. 112 patients ( 51 children with and 61 children without ADHD) between 7 and 17 years old were compared regarding IAD, IGD, and MPA. We used the TEA questionnaire for the assessment of executive function and ADHD (ATENTO), and the ADITEC questionnaire to get gender-differentiated information for IAD, IGD, and MPA. Female children scored higher on MPA (Mean \pm Standard Deviation, $\mathrm{M} \pm \mathrm{SD}$ ) $(25.93 \pm 17.64$ vs. $14.77 \pm 19.43, \mathrm{p}=0.03)$, while male children scored higher on IGD $\left(30.09 \pm 21.65\right.$ vs. $\left.12.51 \pm 16.61, \mathrm{p}<10^{-3}\right)$. Severity of hyperactivity/impulsivity and IGD were moderately correlated $(r=0.349$, $\mathrm{p}=0.013$ ), but the correlation disappeared after controlling for the impact on the social domain as measured by the ATENTO questionnaire $(\mathrm{r}=171, \mathrm{p}=0.250)$. Most parents are concerned that their children may be addicted to IAD/IGD/MPA. Female gender is associated with MPA, while male gender is associated with IGD. ADHD is a risk factor for developing IAD and IGD. Combined type and predominantly hyperactive/impulsive ADHD are each associated with IGD. Good social adjustment protects against developing IGD. There are gender vulnerabilities for IAD/IGD/MPA. ADHD is a risk factor for IGD, but good social adjustment buffers this association.

Keywords: ADHD; Internet addiction disorder; Internet gaming disorder; mobile phone addiction. 


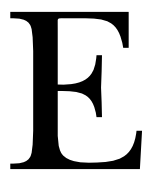

1 uso de las nuevas tecnologías (NNTT) (Internet, teléfonos móviles y videojuegos) se ha generalizado a nivel mundial. Las NNTT son herramientas útiles que facilitan la comunicación y el intercambio de información y que son utilizables para fines de ocio. Sin embargo, un uso excesivo de las NNTT se está convirtiendo cada vez más en un motivo de preocupación (Marin Vila, Carballo Crespo y Coloma Carmona, 2018). Por ejemplo, el $25 \%$ de los jóvenes dedica más de tres horas diarias a ver la televisión o utilizar Internet, y el 15\% dedica más de tres horas a jugar videojuegos en un día escolar (Weiss, Baer, Allan, Saran y Schibuk, 2011). A pesar de ser objeto de controversia (Weinstein, Yaacov, Manning, Danon y Weizman, 2015), hay cada vez más pruebas de que el trastorno por adicción a Internet (TAI), el trastorno de juego en Internet (TJI) y la adicción al teléfono móvil (ATM) son adicciones conductuales (Chóliz, 2010; Osborne et al., 2016; Rehbein, Kliem, Baier, Mossle y Petry, 2015). De hecho, la quinta edición del Manual Diagnóstico y Estadístico de los Trastornos Mentales (DSM-5) incluyó «adicción al juego con dinero en Internet» en el capítulo Trastornos relacionados con sustancias y trastornos adictivos. Además, el TAI y el TJI se incluyeron como condiciones para estudios posteriores en la Sección 3 (Chen, Chen y Gau, 2015; Rehbein et al., 2015).

Existen varios factores de riesgo para el TAI, el TJI y la ATM. Por ejemplo, ciertas características como la extraversión, la desinhibición, el narcisismo, el neuroticismo, la ansiedad social, el apego ansioso y la baja autoestima se asocian con un mayor riesgo de TAI (Peris, Maganto y Garaigordobil, 2018). El TJI se asocia con el género masculino (Schou Andreassen et al., 2016). La ATM se ha relacionado con baja autoestima, ansiedad social y sensibilidad interpersonal (You, Zhang, Zhang, Xu y Chen, 2019).

El trastorno por déficit de atención con hiperactividad (TDAH), el trastorno más frecuente en la infancia y adolescencia con una prevalencia mundial de 5,29\% (rango 0,2\%-27\%) (Polanczyk, de Lima, Horta, Biederman y Rohde, 2007) se ha asociado con el TAI (Dalbudak y Evren, 2014) y el TJI (Weinstein y Weizman, 2012). Algunos estudios no informaron diferencias significativas en la cantidad de tiempo o frecuencia de juego entre niños con y sin el TDAH, sino más bien una mayor severidad de la adicción en el TJI en niños con el TDAH (Bioulac, Arfi y Bouvard, 2008). Sin embargo, hay menos evidencia sobre la relación entre el TDAH y la ATM.

El objetivo principal de este estudio es explorar la relación entre el TDAH y el TAI, el TJI y la ATM en niños y adolescentes. Nuestro estudio tiene tres objetivos específicos: primero, comparar el patrón de uso de las NNTT (Internet, videojuegos, y teléfono móvil) entre niños y adolescentes con y sin el TDAH; segundo, estudiar la relación entre el TDAH y el TAI, el TJI y la ATM; y tercero, explorar si las habilidades sociales median o no en la relación entre el TDAH y el TAI, el TJI y la ATM.

\section{Métodos}

\section{Participantes}

Este estudio es descriptivo naturalista de casos y controles. La muestra fue reclutada en el Hospital Universitario Puerta de Hierro en Majadahonda (HUPH-M). Se reclutaron ciento doce pacientes entre 7-17 años: 51 niños y adolescentes con diagnóstico de TDAH (casos) y 61 niños y adolescentes que asistieron a consultas de pediatría ambulatorias en el mismo hospital por un motivo médico y sin diagnóstico de TDAH (controles). La mayoría de los controles fueron pacientes por razones digestivas o respiratorias. Ni los casos ni los controles presentaron comorbilidad con retraso mental, trastornos generalizados del desarrollo $\mathrm{u}$ otras alteraciones neurológicas o psiquiátricas que pudieran comprometer el funcionamiento cognitivo del participante.

\section{Medidas y procedimiento}

Los casos y controles se evaluaron por medio de entrevistas semiestructuradas de diagnóstico, incluyendo entrevistas parentales y un protocolo que incluyó datos sociodemográficos y clínicos; los criterios de TDAH del Manual diagnóstico y estadístico de los trastornos mentales, $4^{\mathrm{a}}$ ed. (DSM-IV); y algunas escalas para evaluar el TDAH y el TAI, el TJI y la ATM.

Según estos criterios, se dividieron los casos en los siguientes grupos: tipo combinado (TDAH/C), tipo predominantemente hiperactivo/impulsivo (TDAH/HI) y tipo predominantemente inatento (TDAH/I). Para evaluar los síntomas principales del TDAH (impulsividad, hiperactividad e inatención) y los procesos ejecutivos subyacentes alterados, utilizamos el Cuestionario TEA para la evaluación de la función ejecutiva y el TDAH (ATENTO), que evalúa los siguientes aspectos: control de la atención, regulación conductual, regulación emocional, flexibilidad, memoria de trabajo, planificación y organización, orientación temporal, problemas conductuales, problemas relacionados con el sueño, así como el nivel de afectación o impacto de los síntomas en los contextos familiares, sociales y escolares (Sánchez-Sánchez y Luque, 2020).

Evaluamos el uso de las NNTT en base a la información proporcionada por los padres y niños. Los padres participantes completaron un cuestionario ad hoc de 12 ítems (sí/ no) basado en una versión adaptada de los criterios utilizados para la evaluación de las adicciones conductuales (Blasco-Fontecilla et al., 2014) (ver material complementario, MC). Esta estrategia se ha utilizado en otros estudios (Kourosh, Harrington y Adinoff, 2010). Utilizamos la primera pregunta del cuestionario ad hoc para evaluar el uso de las NNTT $\left(_{i}\right.$ Cree que su hijo tiene una adicción a alguna de las siguientes tecnologías?). Si el padre respondió «sí» a tres o más preguntas (ítems 2 a 12), lo consideramos un «diagnóstico de cribado» para la adicción a las NNTT. Con respecto a los niños y adolescentes, utilizamos el cuestionario ADITEC (ADITEC; Evaluación y prevención de la adicción a 
Internet, móvil y videojuegos; Chóliz, Marco y Chóliz, 2016) para diagnosticar el TAI, el TJI y/o la ATM. El cuestionario ADITEC proporciona información diferenciada por género para el TAI, el TJI y la ATM, incluso una puntuación total y las siguientes puntuaciones en las subescalas: 1) Internet (ADITEC-I): abuso, abstinencia, perturbación y ausencia de control y escape; 2) Videojuegos (ADITEC-V): juego compulsivo, abstinencia, tolerancia e interferencia con otras actividades, y problemas asociados y escape; y 3) Teléfono móvil (ADITEC-M): tolerancia y abstinencia, dificultad para controlar el impulso, problemas derivados del gasto económico y abuso (Chóliz, Marco y Chóliz, 2016).

\section{Análisis estadístico}

Para el Objetivo 1, realizamos análisis descriptivos de variables sociodemográficas y clínicas y la frecuencia de adicción a las NNTT en los grupos de casos y controles, utilizando $\chi 2$ y la razón de momios (RM) para variables ordinales e internas. También utilizamos la prueba $t$ para muestras independientes asociadas con la estadística de Levene para la igualdad de varianzas para comparar variables cuantitativas (casos vs. controles, y TDAH/HI vs. TDAH/I). Para evaluar las diferencias entre grupos respecto del TAI, el TJI, y la ATM, realizamos el procedimiento ANOVA bifactorial al diferenciar ambos grupos de edad: 7-11 años y 12-17 años. Así, consideramos el grupo (casos vs. controles) como factor entre-sujeto y el TAI, el TJI y la ATM como factor intra-sujeto. Esta estrategia nos permitió extraer información entre grupos, tipos de adicción a las
NNTT (TAI, TJI y ATM) y la interacción entre el TDAH y el TAI, el TJI y la ATM. Para el Objetivo 2, realizamos un análisis correlacional bivariable para comparar las puntuaciones de las tres subescalas del cuestionario ADITEC (teléfono móvil, videojuegos e Internet) y las puntuaciones de inatención e hiperactividad-impulsividad en base a las mediciones del cuestionario ATENTO. Para el Objetivo 3 , realizamos los mismos análisis correlacional bivariables, pero controlamos la subescala de afectación del contexto social del cuestionario ATENTO. El nivel de significancia estadística se estableció en $p<, 05$. Utilizamos el software SPSS (v20 para Mac) para todos los análisis.

\section{Ética}

Todos los procedimientos del estudio se implementaron según la Declaración de Helsinki. El Comité de Ética local aprobó el estudio (12 de febrero de 2018; n. ${ }^{\circ}$ 03.18). Los padres (o tutores legales) y los niños fueron ampliamente informados sobre el estudio antes de participar en el mismo. Tanto los padres (o tutores legales) como los niños fueron informados y aceptaron participar voluntariamente. Se obtuvieron los consentimientos informados tanto de los padres padres como de los niños antes de participar en este estudio.

\section{Resultados}

La Tabla 1 muestra las características sociodemográficas y educativas, y algunos antecedentes clínicos y familiares, de los participantes.

Tabla 1. Características sociodemográficas y clínicas de los pacientes.

\begin{tabular}{|c|c|c|c|c|}
\hline & $\begin{array}{c}\text { Casos } \\
\mathrm{n}=\mathbf{5 1} \\
\%\end{array}$ & $\begin{array}{c}\text { Controles } \\
n=61 \\
\%\end{array}$ & RM $[95 \%$ IC $]$ & Significancia \\
\hline Género (mujer) & 21,6 & 49,2 & $0,48[0,28-0,82]$ & $\mathrm{FET}^{\star} \mathrm{p}=, 003$ \\
\hline Adoptado (Sî) & 15,7 & 0,0 & $2,42[1,92-3,04]$ & FET $p=, 001$ \\
\hline Repetición de al menos un curso escolar & 28 & 9,8 & $1,77[1,20-2,60]$ & FET $p=, 024$ \\
\hline Apoyo docente en la escuela (Sî) & 37,5 & 13,1 & $1,92[1,31-2,81]$ & FET $p=, 006$ \\
\hline Adaptación del currículo de infancia temprana en la escuela (Sî) & 31,2 & 9,8 & $1,91[1,30-2,79]$ & FET $p=, 007$ \\
\hline Apoyo docente en el hogar (Sî́) & 39,6 & 24,6 & & FET $p=, 101$ \\
\hline Problemas durante el embarazo (Sî́) & 23,8 & 18 & & FET $p=, 619$ \\
\hline Nacimiento (semanas) & & & & FET $p=, 841$ \\
\hline$\langle 370\rangle 42$ & 34,6 & 31,1 & & \\
\hline $37-42$ & 65,4 & 68,9 & & \\
\hline Problemas durante el nacimiento (Sî) & 20,9 & 29,5 & & FET $p=, 370$ \\
\hline Adquisición del habla (> 2 años de edad) & 48,1 & 29,5 & $1,52[1,02-2,22]$ & FET $p=, 053$ \\
\hline Control de esfínteres (> 6 años de edad) & 25 & 6,6 & $1,88[1,32-2,69]$ & FET $p=, 008$ \\
\hline Antecedentes familiares de trastornos mentales & 47,1 & 11,7 & $2,17[1,59-3,33]$ & FET $p<10-3$ \\
\hline
\end{tabular}

Nota. * FET (prueba exacta de Fisher). 


\section{Objetivo 1: Comparar el patrón de uso de las NNTT (Internet, videojuegos y teléfono móvil) en casos versus controles}

El $75,6 \%$ y el $73 \%$ de los padres de casos y controles, respectivamente, respondieron afirmativamente a la primera pregunta del cuestionario ad hoc que evaluaba el uso de las NNTT ${ }_{(i}$ Cree que su hijo tiene una adicción a alguna de las siguientes tecnologías?). Incluso si no encontramos diferencias estadísticamente significativas en cuanto al uso de Internet/videojuegos/teléfono móvil, los padres de niños y adolescentes con el TDAH parecían preocupados particularmente sobre el uso de Internet, mientras que los padres de los controles expresaron su preocupación por el uso del teléfono móvil (véase Tabla 1-MC).

Al utilizar el diagnóstico operativo de adicción a las NNTT ( ${ }^{3} 3$ o más respuestas afirmativas a las preguntas $2-12$ ), el $66,7 \%$ y el $29,7 \%$ de los padres de casos de TDAH y de controles, respectivamente, cumplieron con los criterios de diagnóstico [Relación de probabilidades, RP (95\%) $=2(1,28-3,12)$, prueba exacta de Fisher $($ FET $) p=, 002]$ (véase Figura 1-MC y Tabla 2-MC).

La Figura 1 muestra gráficamente la prevalencia de al menos una adicción a las NNTT (el TAI, el TJI y la ATM). La prevalencia oscila entre el 29,7\% entre los controles pediátricos y el $100 \%$ del subtipo predominantemente $\mathrm{TDAH} / \mathrm{HI}(\mathrm{RP}=3,42[1,23-9,55], \operatorname{FET} p=, 003)$.

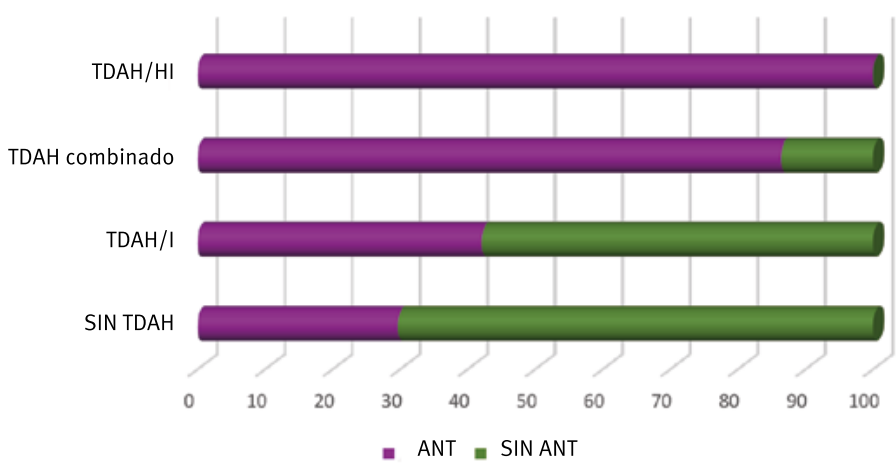

Figura 1. Prevalencia de al menos una «adicción a las nuevas tecnologías» (ANNTT): (TAI/TJI/ATM).

El TDAH se asoció con el TAI y el TJI, pero no con la ATM (véase Tabla 2).

Tabla 2. Comparación entre casos y controles entre el TAl, el TJl y la ATM (medido por las respuestas directas en el ADITEC).

\begin{tabular}{lcccc}
\hline & $\begin{array}{c}\text { Casos } \\
(\mathbf{n}=\mathbf{5 1}) \\
\mathbf{M} \pm \mathbf{D T}\end{array}$ & $\begin{array}{c}\text { Controles } \\
(\mathbf{n}=\mathbf{6 1})\end{array}$ & & \\
\hline ATM & $18,86 \pm 19,20$ & $19,15 \pm 19,77$ & 0,794 & $\mathrm{p}=, 939$ \\
TII & $32,10 \pm 23,70$ & $16,79 \pm 16,89$ & 0,001 & $\mathrm{p}<, 001$ \\
TAI & $26,94 \pm 21,18$ & $17,70 \pm 17,23$ & 0,079 & $\mathrm{p}=, 013$ \\
\hline
\end{tabular}

La Figura 2 muestra las diferencias cuantitativas entre el TAI, el TJI y la ATM (medido por las respuestas directas en el ADITEC) por género. Las niñas obtuvieron puntuaciones más altas en la ATM (desviación típica \pm media, DT $\pm M)(25,93 \pm 17,64$ vs. $14,77 \pm 19,43, p=, 03)$ y en el TAI $(23,59 \pm 18,72$ vs. $20,68 \pm 20,22, p=, 45)$, mientras que los niños obtuvieron puntuaciones más altas en el TJI $(30,09 \pm$ 21,65 vs. $\left.12,51 \pm 16,61, p<10^{-3}\right)$.

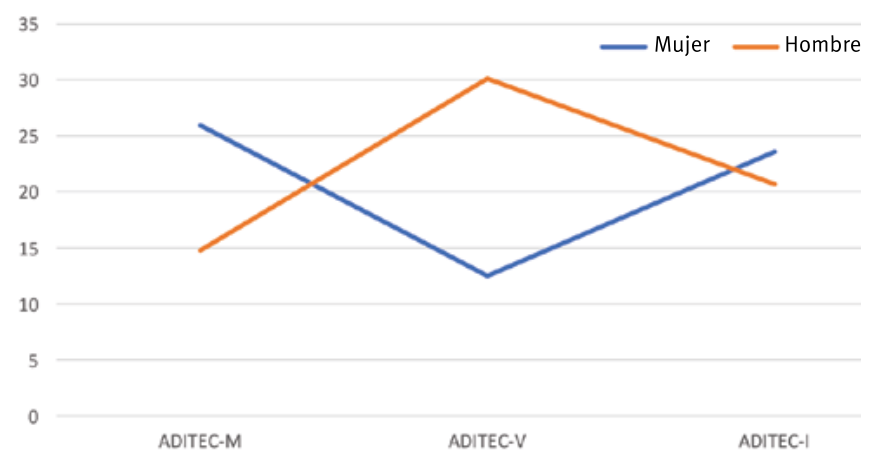

Figura 2. Tipo de ANNTT (medido por el ADITEC*: TAI/TJI/ATM) por género.

Nota. *ADITEC-M (Móvil); ADITEC-V (Videojuegos); ADITEC-I (Internet).

\section{Objetivo 2: Estudiar la relación entre el TDAH y la adicción a las NNTT (ANNTT)}

La Tabla 3 muestra la relación entre los diversos subtipos del TDAH y el TAI, el TJI y la ATM.

Encontramos una correlación positiva entre la severidad de la impulsividad y el TJI (véase Tabla 4) En consecuencia, exploramos cuáles de las subescalas del TJI estaban involucradas. Descubrimos que la gravedad de H/I se correlacionaba tanto con la abstinencia como con los problemas asociados y las subescalas de escape (véase Tabla 5).

\section{Objetivo 3: Probar la influencia del contexto social en la ANNTT}

Después de controlar por el impacto del dominio social según las mediciones del cuestionario ATENTO, las correlaciones mostradas en la Tabla 6 fueron inferiores que las mostradas en la Tabla 4 y ya no fueron estadísticamente significativas.

Tabla 3. Subtipos de TDAH (DSM-IV) y el TAl, el TJl y la ATM (medido por las respuestas directas en el ADITEC*).

\begin{tabular}{lcccc}
\hline & $\begin{array}{c}\text { TDAH/I } \\
(\mathbf{n}=\mathbf{2 4})\end{array}$ & $\begin{array}{c}\text { TDAH/HI o el } \\
\text { TDAH/C }(\mathbf{n}=\mathbf{2 6})\end{array}$ & & \\
& $\mathrm{M} \pm \mathrm{DT}$ & $\mathrm{M} \pm \mathrm{DT}$ & Levene & Prueba $\mathbf{~}$ \\
\hline ATM & $22,75 \pm 19,25$ & $14,73 \pm 18,88$ & 0,794 & $\mathrm{p}=, 144$ \\
TJI & $22,79 \pm 18,91$ & $40,31 \pm 25,25$ & 0,201 & $\mathrm{p}=, 008$ \\
TAI & $27,13 \pm 22,33$ & $26,75 \pm 20,91$ & 0,865 & $\mathrm{p}=, 952$ \\
\hline
\end{tabular}


Tabla 4. Correlaciones entre las dimensiones del TDAH (medido por el cuestionario ATENTO) y el TAl, el TJl y la ATM (medido por el cuestionario ADITEC).

\begin{tabular}{llccc}
\hline & & ATM & TJI & TAI \\
\hline \multirow{2}{*}{ Inatención } & r de Pearson &,- 145 &, 219 &, 007 \\
\cline { 2 - 5 } & Significancia &, 316 &, 127 &, 961 \\
\hline $\begin{array}{l}\text { Hiperactividad/ } \\
\text { Impulsividad }\end{array}$ & r de Pearson &,- 079 &, 349 &, 066 \\
\cline { 2 - 5 } & Significancia &, 586 &, 013 &, 657 \\
\hline
\end{tabular}

Tabla 5. Correlaciones entre las dimensiones del TDAH (medido por el cuestionario ATENTO) y las subescalas del TJI.

\begin{tabular}{lccccc}
\hline \multirow{2}{*}{ Inatención } & & $\begin{array}{c}\text { Juego } \\
\text { compulsivo }\end{array}$ & $\begin{array}{c}\text { Absti- } \\
\text { nencia }\end{array}$ & $\begin{array}{c}\text { Tolerancia } \\
\text { e inter- } \\
\text { ferencia }\end{array}$ & $\begin{array}{c}\text { asoblemas } \\
\text { y escape }\end{array}$ \\
\cline { 2 - 6 } & rde Pearson &, 164 &, 219 &, 211 &, 239 \\
\hline \multirow{2}{*}{$\begin{array}{l}\text { Hipneractividad/cancia } \\
\text { Impulsividad }\end{array}$} &, 254 &, 126 &, 142 &, 095 \\
\cline { 2 - 6 } & Significancia &, 056 &, 008 &, 072 &, 004 \\
\hline
\end{tabular}

Tabla 6. Correlaciones entre el TDAH y el TAl, el TJI y la ATM, controlando por la subescala Afectación del contexto social (ATENTO).

\begin{tabular}{llccc}
\hline & & ADITEC-M & ADITEC-V & ADITEC-I \\
\hline \multirow{2}{*}{ Inatención } & rde Pearson &, 005 &,- 074 &,- 184 \\
\cline { 2 - 5 } & Significancia &, 972 &, 619 &, 217 \\
\hline $\begin{array}{l}\text { Hiperactividad/ } \\
\text { Impulsividad }\end{array}$ & rde Pearson &, 051 &, 171 &,- 063 \\
\cline { 2 - 5 } & Significancia &, 734 &, 250 &, 674 \\
\hline
\end{tabular}

\section{Discusión}

De acuerdo con la literatura, nuestro estudio confirmó que hay una prevalencia alta de adicción a las NNTT (el TAI, el TJI y la ATM) entre niños y adolescentes con y sin el TDAH. También encontramos que el TDAH está asociado con el TAI, el TJI y la ATM en una población española de niños y adolescentes. La asociación entre el TDAH y el TAI, el TJI y la ATM se ha demostrado de manera convincente en varios países asiáticos (Lee et al., 2018), pero faltan pruebas en otros países. Además, ampliamos cierta información sobre la relación entre el TDAH y el TAI, el TJI y la ATM. Por ejemplo, encontramos que el TDAH/C y el TDAH/HI estaban asociados con el TJI, mientras que el TDAH/I estaba relacionado con la ATM. Sin embargo, el hallazgo más relevante fue que un buen ajuste social amortiguó la relación entre el TDAH/C y el TDAH/HI y el TJI en niños y adolescentes con el TDAH.

Al utilizar nuestros criterios ad hoc para diagnosticar una adicción a las NNTT, encontramos que la mayoría de los padres de niños y adolescentes con o sin el TDAH temían que pudieran tener al menos una adicción a las NNTT (el TAI, el TJI y la ATM). Estos porcentajes elevados pueden sugerir que el umbral utilizado ( ${ }^{3} 3$ ítems) era demasiado bajo para usar estos criterios como herramienta de cribado. En cualquier caso, la probabilidad de comorbilidad del TAI, el TJI y la ATM es muy alta. Por tanto, el TAI oscila entre el 1,4\% y 17,9\% de los adolescentes (Chen et al., 2015), llegando al 21,6\% en Corea (Lee et al., 2018). Además, el 8\% de las personas de 17 o menos años son diagnosticadas con el TJI (Stockdale et al., 2018); el 36,6\% de los adultos con el TDAH y el $23 \%$ de los controles sin el TDAH tenían al menos una adicción a las NNTT (Bielefeld et al., 2017).

Por otro lado, encontramos una diferencia estadísticamente significativa de género entre los casos y los controles, ya que el género masculino estaba sobrerrepresentado entre el grupo con TDAH. De hecho, encontramos una relación hombre:mujer de 3:1, que es la relación hombre:mujer aceptada en la literatura (Lange, Reichl, Lange, Tucha y Tucha, 2010). Es importante destacar esta diferencia de género, ya que puede afectar nuestros resultados. De hecho, ambos géneros femenino y masculino se asociaron con la ATM y el TJI, respectivamente (Haghbin, Shaterian, Hosseinzadeh y Griffiths, 2013; Schou Andreassen et al., 2016). Estas diferencias de género pueden reflejar la propensión de los chicos a participar en entretenimientos competitivos y agresivos y de las chicas a participar en actividades cooperativas y sociales (Griffiths, Kuss y Pontes, 2016).

De acuerdo con la literatura, encontramos que el TDAH es un factor de riesgo para el TAI (Dalbudak y Evren, 2014) y el TJI (Weinstein y Weizman, 2012), pero no para la ATM. Nuestros hallazgos son particularmente interesantes porque el TAI no estaba asociado con ningún subtipo particular de TDAH, pero el TJI estaba claramente asociado con el TDAH/C y el TDAH/HI. La mayoría de los autores han informado que la impulsividad es fundamental para la adicción a las NNTT. En un estudio, la impulsividad fue el predictor más relevante de desarrollar el TAI (Metin et al., 2015). Estos autores enfatizaron que los niños con mayor probabilidad de tener comorbilidad con el TAI comparten una preferencia por estímulos de respuesta rápida y recompensas a corto plazo, desinhibición y falta de autocontrol e impulsividad. Esta opinión, junto con la evidencia de que las personas con el TAI tienen déficits para inhibir las respuestas a los estímulos en condiciones experimentales (Cao, Su, Liu y Gao, 2007), ha llevado a algunos autores a proponer el TAI como un trastorno de control de impulsos (Cao et al., 2007; Metin et al., 2015). Además, un metaanálisis reciente concluyó que la impulsividad mediaba la relación entre el TDAH y la adicción a los medios (Nikkelen, Valkenburg, Huizinga y Bushman, 2014).

Nuestro hallazgo que el TDAH/C y el TDAH/HI son los subtipos más estrechamente asociados con el TJI es consistente con un estudio que postula que la impulsividad explica la relación entre autocontrol, el TDAH y el TJI (Haghbin et al., 2013). De hecho, las personas con el TJI tienen disfunciones neurocognitivas y cognitivosociales 
similares a las de los pacientes dependientes de metanfetamina (Jiang, Li, Zhou y Zhou, 2020). Además, los videojuegos proporcionan recompensas rápidas e inmediatas y un entorno de vida artificial donde los niños y adolescentes pueden escapar de los problemas y cumplir sus fantasías (Gentile, Swing, Lim y Khoo, 2012). Es más, la gravedad de $\mathrm{H} / \mathrm{I}$ se correlacionaba tanto con la abstinencia como con los problemas asociados y las subescalas de escape. Este hallazgo es relevante porque apunta a áreas específicas de adicción que podrían ser específicamente dirigidas para el tratamiento si nuestros hallazgos se replican. No hemos encontrado literatura alguna sobre este tema. También encontramos una tendencia de una asociación clínicamente relevante entre el TDAH/I y la ATM. Este hallazgo es similar al reportado en un estudio realizado con adolescentes chinos con TDAH/I, que eran particularmente vulnerables al desarrollo de la ATM si el teléfono móvil se encendía durante el sueño (Zheng et al., 2014).

Sin embargo, el hallazgo más importante de nuestro estudio es que un buen ajuste social amortiguó la relación entre el TDAH/C o el TDAH/HI. Este hallazgo es importante porque es escasa la investigación sobre el impacto del uso de los ordenadores y videojuegos en el desarrollo de las habilidades sociales de niños y adolescentes (Tran y Subrahmanyam, 2013). Por tanto, debemos enfrentar el dilema del huevo y la gallina: ¿El TJI empeora las habilidades sociales? ¿O las personas con habilidades sociales pobres tienden a usar videojuegos? ¿O pueden ambas hipótesis ser ciertas? En un estudio de casos y controles, los estudiantes con el TJI tenían habilidades sociales más pobres que los estudiantes que no tenían el TJI (Zamani, Kheradmand, Cheshmi, Abedi y Hedayati, 2010). En un estudio longitudinal, un seguimiento de dos años de 251 niños midió el impacto del tiempo de pantalla (juegos en la televisión/ videojuegos, videojuegos/juegos en el ordenador) y la actividad física en el desarrollo de las habilidades sociales. Informaron que el tiempo de pantalla se asoció negativamente con las puntuaciones express (p. ej., se une al juego) y de cumplimiento (p. ej., coopera) y positivamente con las puntuaciones disruptivas (p. ej., se burla) (Carson et al., 2019). De manera similar, en otro estudio los autores informaron una relación inversa entre la actividad física (fitness y culturismo) y los problemas de autorregulación relacionados con los videojuegos (Cardol, Escamilla, Gebhardt y Perales, 2019).

Además, la hipótesis de la automedicación puede ayudar a explicar por qué las personas que carecen de habilidades sociales hacen un uso masivo de los videojuegos. En un estudio con 62 niños, sin tratamiento farmacológico previo, diagnosticados con el TDAH y jugadores de videojuegos en Internet, los autores informaron que después de ocho semanas de tratamiento con metilfenidato, los niños no solo mejoraron sus síntomas de TDAH, sino que también redujeron el tiempo que pasaron jugando videojuegos en Internet (Han et al., 2009). Los autores recordaron que tanto los estimulantes -como el metilfenidato- administrados para tratar el TDAH como jugar a videojuegos aumentan la dopamina sináptica, y sugerían que «jugar videojuegos en Internet podría ser una forma de automedicación para niños con el TDAH». De hecho, aunque suele percibirse negativamente la influencia del uso de videojuegos sobre la salud mental de los niños, un estudio reciente con 3.195 niños con edades de 6 a 11 años de seis países europeos informó que el uso elevado de videojuegos estaba relacionado con 1,75 veces mayores probabilidades de un alto funcionamiento intelectual y una disminución del riesgo de problemas de relación entre pares y déficits prosociales [RM 0,41 (0,2-0,86) y 0,23 (0,07, 0,81), respectivamente] (Kovess-Masfety et al., 2016). En un ensayo aleatorio con 69 niños de 7-11 años con habilidades sociales deficientes, 33 fueron asignados al tratamiento con un juego interactivo de aventuras en línea durante nueve semanas y 36 a una lista de espera. El grupo de tratamiento mejoró, de manera estadísticamente significativa, más que los controles «en alfabetización social, ansiedad social, victimización por acoso y satisfacción social» (Sánchez, Brown, Kocher y DeRosier, 2017). Los autores concluyeron que los videojuegos pueden ser útiles para mejorar la salud mental de los niños con habilidades sociales deficientes. En consecuencia, parece que el uso de videojuegos podría ser útil en niños y adolescentes con o sin el TDAH que tienen problemas en sus habilidades sociales.

Se deben mencionar algunas limitaciones de nuestro estudio. Primero, este estudio es retrospectivo. Por tanto, el sesgo del recuerdo puede afectar nuestros resultados y no pueden establecerse vínculos causales. En segundo lugar, el tamaño muestral implicó que algunos resultados clínicamente significativos (p. ej., la tendencia de los padres de niños con el TDAH a estar más preocupados por el uso de Internet que los padres de los controles) no alcanzaran significación estadística. Otra limitación es que el grupo de control se extrajo de pacientes que asistieron a una consulta pediátrica. Por tanto, los resultados epidemiológicos pueden no ser representativos de la población general de niños y adolescentes en nuestro país. Otra limitación es nuestra utilización de un cuestionario ad hoc con los padres para evaluar la percepción del uso de las NNTT por parte de sus hijos; el cuestionario se basó en los criterios modificados del DSM-IV-TR para la dependencia de sustancias. Sin embargo, esta estrategia se ha utilizado para evaluar otras adicciones conductuales, la adicción a las conductas suicidas o al bronceado (Blasco-Fontecilla et al., 2014; Kourosh et al., 2010). Por último, utilizamos cuestionarios nuevos disponibles solo en español para evaluar la gravedad del TDAH y la adicción a las NNTT (ATENTO y ADITEC, respectivamente). Sin embargo, el cuestionario ATENTO es de interés particular porque evalúa no solo los 
síntomas clínicos centrales del TDAH sino también la función ejecutiva y el impacto de los síntomas del TDAH en varios ámbitos, incluido el social. A pesar de su novedad, el cuestionario ADITEC amplía la información sobre varios aspectos de los comportamientos adictivos y puede ser útil para expandir nuestro conocimiento de áreas específicas (p. ej., impulsividad, tolerancia) que se alteran en el TAI, el TJI y la ATM. Además, el ADITEC evalúa exhaustivamente el TAI, el TJI y la ATM en un solo cuestionario.

\section{Conclusiones}

A la mayoría de los padres les preocupa que sus hijos puedan tener adicción a las NNTT (el TAI, el TJI y la ATM). El género femenino está asociado con la ATM, mientras que el género masculino está asociado con el TJI. El TDAH es un factor de riesgo para el desarrollo del TAI y el TJI. El TDAH/C y el TDAH/HI están cada uno asociado con el TJI. Un buen ajuste social protege contra el desarrollo del TJI. Las direcciones futuras para la investigación pueden incluir, entre otras: 1) el estudio del buen ajuste social como factor protector contra el desarrollo de un TJI; 2) probar si el uso de deportes o algunos videojuegos puede proteger contra el desarrollo un TJI; y 3) el estudio de cómo algunas características de los videojuegos (i.e., la cantidad de tiempo, la frecuencia de juego, la presencia o ausencia de violencia, etc.) interactúan con las características de personalidad y el género de los niños y adolescentes (López-Fernández, Mezquita, Griffiths, Ortet y Ibáñez, 2021), para que podamos desarrollar reglas preventivas destinadas a proteger a niños y adolescentes vulnerables.

\section{Reconocimientos}

Los autores agradecen a Lorraine Maw, MA, por su asistencia editorial en su versión en inglés.

\section{Conflicto de intereses}

En los últimos 24 meses, Hilario Blasco-Fontecilla recibió honorarios de Shire por ser conferenciante. Es investigador principal (IP) de un contrato de investigación iPFIS (www.isciii.es; IFI16/00039) y co-IP de una subvención de investigación MINECO (RTI2018-101857-B-I00); beneficiario de: 1) una beca FIPSE, y 2) una beca de intensificación IDIPHIPSA; participante en dos ensayos clínicos (MENSIA KOALA, NEWROFEED Study; ESKETSUI2002); miembro del Consejo Asesor de ITA Salud Mental. Fernando Sánchez es empleado de TEA ediciones. María Rodrigo Yanguas es beneficiaria de un contrato de investigación iPFS (www.isciii.es). Los autores restantes no tienen ningún conflicto de intereses respecto de la publicación de este manuscrito.

\section{Ayudas económicas}

Este estudio no ha sido beneficiario de ningún apoyo financiero ni patrocinio indirecto.

\section{Contribuciones de los autores}

Todos los autores han participado suficientemente en el trabajo para asumir la responsabilidad pública por su contenido. El autor contacto principal del manuscrito afirma que tuvo acceso a todos los datos del estudio, tanto lo que se informa como lo que no se informa, y también tenía total libertad para dirigir su análisis e informes.

H.B.F. concibió y diseñó el estudio. A.M. y A.J.A. recopilaron todos los datos clínicos y de protocolo. M.R.Y. diseñó la base de datos e introdujo la mayoría de los datos.

H.B.F. y A.M. estuvieron a cargo del análisis de datos, las búsquedas bibliográficas, la redacción y revisión de las versiones iniciales del manuscrito.

F.S.S., E.R.R. y M.M.V. revisaron el manuscrito y ofrecieron orientación conceptual para la mejora del estudio.

Todos los autores leyeron, revisaron críticamente y aprobaron la versión definitiva del manuscrito; no se han omitido otros autores potenciales de la autoría.

\section{Ética}

El Comité de Ética del Hospital Universitario Puerta de Hierro-Majadahonda aprobó el estudio (12 de febrero de 2018; n. $\left.{ }^{\circ} 03.18\right)$.

\section{Referencias}

Bielefeld, M., Drews, M., Putzig, I., Bottel, L., Steinbuchel, T., Dieris-Hirche, J.,... Theodor Te Wildt, B. (2017). Comorbidity of Internet use disorder and attention deficit hyperactivity disorder: Two adult case-control studies. Journal of Behavioral Addictions, 6, 490-504. doi:10.1556/2006.6.2017.073.

Bioulac, S., Arfi, L. y Bouvard, M. P. (2008). Attention deficit/hyperactivity disorder and video games: A comparative study of hyperactive and control children. European Psychiatry, 23, 134-141. doi:S0924-9338(07)01444-7.

Blasco-Fontecilla, H., Artieda-Urrutia, P., Berenguer-Elias, N., Garcia-Vega, J. M., Fernandez-Rodriguez, M., Rodriguez-Lomas, C.,... de Leon, J. (2014). Are major repeater patients addicted to suicidal behavior? Adicciones, 26, 321-333.

Cao, F., Su, L., Liu, T. y Gao, X. (2007). The relationship between impulsivity and Internet addiction in a sample of Chinese adolescents. European Psychiatry, 22, 466-471. doi:10.1016/j.eurpsy.2007.05.004.

Cardol, C. K., Escamilla, C. I., Gebhardt, W. A. y Perales, J. C. (2019). Exploring the direct or inverse association of physical activity with behavioral addictions and 
other self-regulation problems. Adicciones, 31, 18-32. doi:10.20882/adicciones.926.

Carson, V., Lee, E. Y., Hesketh, K. D., Hunter, S., Kuzik, N., Predy, M.,... Hinkley, T. (2019). Physical activity and sedentary behavior across three time-points and associations with social skills in early childhood. BMC Public Health, 19, 27. doi:10.1186/s12889-018-6381-x.

Chen, Y. L., Chen, S. H. y Gau, S. S. (2015). ADHD and autistic traits, family function, parenting style, and social adjustment for Internet addiction among children and adolescents in Taiwan: A longitudinal study. Research In Developmental Disabilities, 39, 20-31. doi:10.1016/j. ridd.2014.12.025.

Chóliz, M. (2010). Mobile phone addiction: A point of issue. Addiction, 105, 373-374.

Chóliz, M., Marco, C. y Chóliz, C. (2016). ADITEC. Evaluación y prevención de la adicción a internet, móvil y videojuegos. Madrid: TEA Ediciones.

Dalbudak, E. y Evren, C. (2014). The relationship of Internet addiction severity with Attention Deficit Hyperactivity Disorder symptoms in Turkish University students; impact of personality traits, depression and anxiety. Comprehensive Psychiatry, 55, 497-503. doi:10.1016/j.comppsych.2013.11.018.

Gentile, D. A., Swing, E. L., Lim, C. G. y Khoo, A. (2012). Video game playing, attention problems, and impulsiveness: Evidence of bidirectional causality. Psychology of Popular Media Culture, 1, 62-70.

Griffiths, M. D., Kuss. D. J. y Pontes, H. M. (2016). A brief overview of Internet gaming disorder and its treatment. Australian Clinical Psychologist, 1, 1-12.

Haghbin, M., Shaterian, F., Hosseinzadeh, D. y Griffiths, M. D. (2013). A brief report on the relationship between self-control, video game addiction and academic achievement in normal and ADHD students. Journal of Behavioral Addictions, 2, 239-243. doi:10.1556/JBA.2.2013.4.7.

Han, D. H., Lee, Y. S., Na, C., Ahn, J. Y., Chung, U. S., Daniels, M. A.,... Renshaw, P. F. (2009). The effect of methylphenidate on Internet video game play in children with attention-deficit/hyperactivity disorder. Comprehensive Psychiatry, 50, 251-256. doi:10.1016/j.comppsych.2008.08.011.

Jiang, C., Li, C., Zhou, H. y Zhou, Z. (2020). Individuals with internet gaming disorder have similar neurocognitive impairments and social cognitive dysfunctions as methamphetamine-dependent patients. Adicciones. Avance de publicación on-line. doi:10.20882/adicciones.1342.

Kourosh, A. S., Harrington, C. R. y Adinoff, B. (2010). Tanning as a behavioral addiction. American Journal of Drug and Alcohol Abuse, 36, 284-290. doi:10.3109/00952990.2 010.491883 .

Kovess-Masfety, V., Keyes, K., Hamilton, A., Hanson, G., Bitfoi, A., Golitz, D.,... Pez, O. (2016). Is time spent playing video games associated with mental health, cognitive and social skills in young children? Social Psychiatry and Psychiatric Epidemiology, 51, 349-357. doi:10.1007/s00127016-1179-6.

Lange, K. W., Reichl, S., Lange, K. M., Tucha, L. y Tucha, O. (2010). The history of attention deficit hyperactivity disorder. Attention Deficit Hyperactivty Disorder, 2, 241-255. doi:10.1007/s12402-010-0045-8.

Lee, J. Y., Kim, S. Y., Bae, K. Y., Kim, J. M., Shin, I. S., Yoon, J. S. y Kim, S. W. (2018). Prevalence and risk factors for problematic Internet use among rural adolescents in Korea. Asia Pac Psychiatry, 10, e12310. doi:10.1111/ appy.12310.

López-Fernández, F. J., Mezquita, L., Griffiths, M. D., Ortet, G. y Ibáñez, M. I. (2021). The role of personality on disordered gaming and game genre preferences in adolescence: Gender differences and person-environment transactions. Adicciones, 33, 263-272. doi:10.20882/adicciones.1370.

Marin Vila, M., Carballo Crespo, J. L. y Coloma Carmona, A. (2018). Academic outcomes and cognitive performance in problematic Internet users. Adicciones, 30, 101110. doi:10.20882/adicciones.844.

Metin, O., Saracli, O., Atasoy, N., Senormanci, O., Kardes, V. C., Acikgoz, H. O.,... Tahiroglu, A. Y. (2015). Association of internet addiction in high school students with ADHD and tobacco/alcohol use. Dusunen Adam, 28, 204-212.

Nikkelen, S. W., Valkenburg, P. M., Huizinga, M. y Bushman, B. J. (2014). Media use and ADHD-related behaviors in children and adolescents: A meta-analysis. Developmental Psychology, 50, 2228-2241. doi: 10.1037/a0037318.

Osborne, L. A., Romano, M., Re, F., Roaro, A., Truzoli, R. y Reed, P. (2016). Evidence for an internet addiction disorder: Internet exposure reinforces color preference in withdrawn problem users. Journal of Clinical Psychiatry, 77, 269-274. doi:10.4088/JCP.15m10073.

Peris, M., Maganto, C. y Garaigordobil, M. (2018). Escala de riesgo de adicción-adolescente a las redes sociales e internet: Fiabilidad y validez (ERA-RSI). Revista de psicología Clínica con Niños y Adolescentes, 5, 30-36.

Polanczyk, G., de Lima, M. S., Horta, B. L., Biederman, J. y Rohde, L. A. (2007). The worldwide prevalence of ADHD: A systematic review and metaregression analysis. American Journal of Psychiatry, 164, 942-948. doi:10.1176/ appi.ajp.164.6.942.

Rehbein, F., Kliem, S., Baier, D., Mossle, T. y Petry, N. M. (2015). Prevalence of Internet gaming disorder in German adolescents: Diagnostic contribution of the nine DSM- 5 criteria in a state-wide representative sample. Addiction, 110, 842-851. doi:10.1111/add.12849.

Sánchez, R., Brown, E., Kocher, K. y DeRosier, M. (2017). Improving children's mental health with a digital social skills development game: A randomized controlled effi- 
cacy trial of adventures aboard the S.S. GRIN. Games Health Journal, 6, 19-27. doi:10.1089/g4h.2015.0108.

Sánchez-Sánchez, F. y Luque, T. (2020). ATENTO, Cuestionario TEA para la evaluación de las funciones ejecutivas y el TDAH. Madrid: TEA Ediciones.

Schou Andreassen, C., Billieux, J., Griffiths, M. D., Kuss, D. J., Demetrovics, Z., Mazzoni, E. y Pallesen, S. (2016). The relationship between addictive use of social media and video games and symptoms of psychiatric disorders: A large-scale cross-sectional study. Psychology of Addictive Behaviors, 30, 252-262. doi:10.1037/adb0000160.

Stockdale, L., Coyne, S.M. (2018). Video game addiction in emerging adulthood: Cross-sectional evidence of pathology in video game addicts as compared to matched healthy controls. Journal of Affective Disorders, 225, 265272. doi:10.1016/j.jad.2017.08.045.

Tran, P. y Subrahmanyam, K. (2013). Evidence-based guidelines for the informal use of computers by children to promote the development of academic, cognitive and social skills. Ergonomics, 56, 1349-1362. doi:10.1080/001 40139.2013.820843.

Weinstein, A. y Weizman, A. (2012). Emerging association between addictive gaming and attention-deficit/hyperactivity disorder. Current Psychiatry Reports, 14, 590-597. doi:10.1007/s11920-012-0311-x.
Weinstein, A., Yaacov, Y., Manning, M., Danon, P. y Weizman, A. (2015). Internet addiction and Attention Deficit Hyperactivity Disorder among schoolchildren. The Israel Medical Association Journal, 17, 731-734.

Weiss, M. D., Baer, S., Allan, B. A., Saran, K. y Schibuk, H. (2011). The screens culture: Impact on ADHD. Attention Deficit Hyperactivity Disorder, 3, 327-334. doi:10.1007/ s12402-011-0065-Z.

You, Z., Zhang, Y., Zhang, L., Xu, Y. y Chen, X. (2019). How does self-esteem affect mobile phone addiction? The mediating role of social anxiety and interpersonal sensitivity. Psychiatry Research, 271, 526-531. doi:10.1016/j. psychres.2018.12.040.

Zamani, E., Kheradmand, A., Cheshmi, M., Abedi, A. y Hedayati, N. (2010). Comparing the social skills of students addicted to computer games with normal students. $A d-$ diction Health, 2, 59-65.

Zheng, F., Gao, P., He, M., Li, M., Wang, C., Zeng, Q.,... Zhang, L. (2014). Association between mobile phone use and inattention in 7102 Chinese adolescents: A population-based cross-sectional study. BMC Public Health, 14, 1022. doi: 10.1186/1471-2458-14-1022. 


\section{Material complementario (MC)}

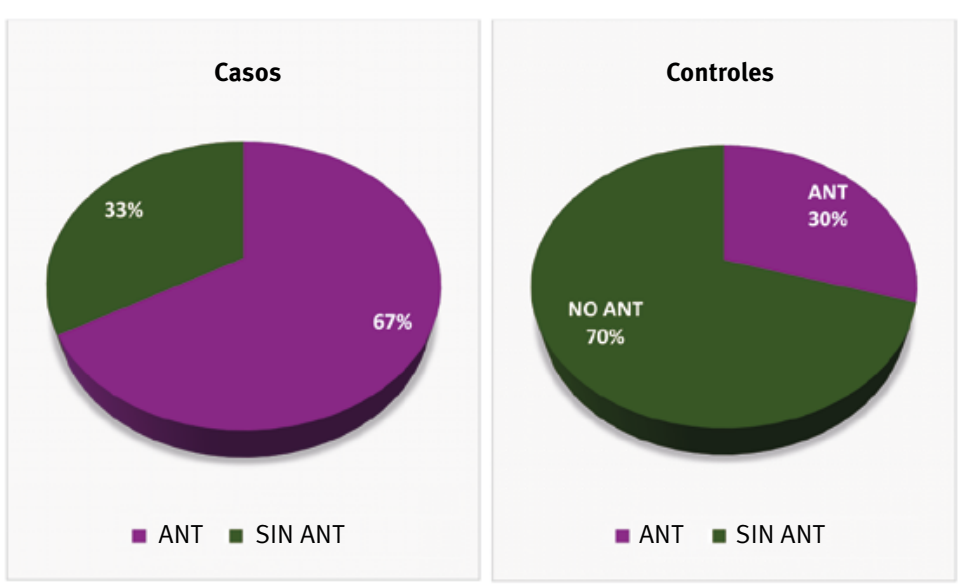

Figura 1-MC. Prevalencia de una adicción a las nuevas tecnologías.

(ANNTT): el TAI, el TJl y/o la ATM) entre casos y controles.

Tabla 1-MC. Percepción de una ANNTT por parte de los padres (respuestas a la pregunta 1 del cuestionario ad hoc).

\begin{tabular}{|c|c|c|c|c|c|c|}
\hline & No & Móvil & Videojuegos & Internet & Varias & Global \\
\hline Casos & $11(24,4 \%)$ & $6(13,1 \%)$ & $14(31,1 \%)$ & $12(26,7 \%)$ & $2(4,4 \%)$ & 45 (100\%) \\
\hline Controles & $10(27 \%)$ & $9(24,3 \%)$ & $12(32,4 \%)$ & $5(13,5 \%)$ & $1(2,7 \%)$ & 37 (100\%) \\
\hline Global & $21(25,6 \%)$ & $15(18,3 \%)$ & $26(31,7 \%)$ & $17(20,7 \%)$ & $3(3,7 \%)$ & 82 (100\%) \\
\hline
\end{tabular}

Tabla 2-MC. Cuestionario ad hoc sobre una ANNTT putativa (padres).

\begin{tabular}{|c|c|c|c|}
\hline Ítem & $\begin{array}{c}\text { Casos } \\
(\%)\end{array}$ & $\begin{array}{l}\text { Controles } \\
(\%)\end{array}$ & Significancia \\
\hline 1. ¿Cree que su hijo/a tiene una adicción a alguna de las siguientes tecnologías? & & & ns \\
\hline $\begin{array}{l}\text { 2. ¿Su hijo/a ha tenido el impulso de usar las nuevas tecnologías (móvil, videojuegos o Internet) } \\
\text { para aliviar la tensión, relajarse o disminuir el sufrimiento psicológico durante el último año? }\end{array}$ & & & ns \\
\hline $\begin{array}{l}\text { 3. ¿Su hijo/a ha estado utilizando las nuevas tecnologías con mayor frecuencia o durante más tiempo } \\
\text { que el inicialmente planificado? }\end{array}$ & & & ns \\
\hline $\begin{array}{l}\text { 4. ¿Su hijo/a tiene el deseo persistente de dejar de utilizar las nuevas tecnologías, } \\
\text { pero es incapaz de dejar de hacerlo? }\end{array}$ & & & ns \\
\hline $\begin{array}{l}\text { 5. ¿Su hijo/a alguna vez ha dejado de asistir o ha interrumpido su participación en un evento social, } \\
\text { trabajo, estudios u otras actividades de ocio debido a su implicación en actividades } \\
\text { relacionadas con las nuevas tecnologías? }\end{array}$ & 46,7 & 21,6 & $p=, 018$ \\
\hline $\begin{array}{l}\text { 6. ¿Su hijo/a continúa utilizando las nuevas tecnologías a pesar de conocer } \\
\text { los problemas relacionados con su utilización? }\end{array}$ & & & ns \\
\hline $\begin{array}{l}\text { 7. ¿Su hijo/a alguna vez ha intentado de dejar de utilizar las nuevas tecnologías, } \\
\text { pero fue incapaz de hacerlo o le requirió un esfuerzo considerable? }\end{array}$ & & & ns \\
\hline $\begin{array}{l}\text { 8. ¿Cree que su hijo/a necesita dedicar cada vez más tiempo a las nuevas tecnologías } \\
\text { con el fin de sentirse bien, disminuir su ansiedad o estabilizar sus emociones? }\end{array}$ & 44,4 & 2,7 & $p<, 05$ \\
\hline $\begin{array}{l}\text { 9. ¿Su hijo/a siente un gran deseo de utilizar las nuevas tecnologías incluso } \\
\text { sin tener una razón específica por hacerlo? }\end{array}$ & 64,4 & 16,2 & $p<, 05$ \\
\hline $\begin{array}{l}\text { 10. ¿Su hijo/a ha tenido algún problema en la escuela/el trabajo/el hogar debido } \\
\text { a las nuevas tecnologías? }\end{array}$ & 42,2 & 0 & $p<, 05$ \\
\hline $\begin{array}{l}\text { 11. ¿Su hijo/a utiliza las nuevas tecnologías en situaciones en las cuales supone un riesgo físico? } \\
\text { (i.e., al cruzar una calle mientras utiliza el móvil) }\end{array}$ & 22,2 & 0 & $p<, 05$ \\
\hline $\begin{array}{l}\text { 12. ¿Su hijo/a se siente mal, ansioso/a o molesto/a cuando desea utilizar las nuevas tecnologías } \\
\text { pero no puede en ese momento? }\end{array}$ & & & ns \\
\hline
\end{tabular}

